\title{
Lower leg compartment syndrome following prolonged orthopedic surgery in the lithotomy position
}

\section{-A case report-}

\author{
Jin Hun Chung ${ }^{1}$, Ki Ryang Ahn ${ }^{1}$, Jin Hyung Park ${ }^{1}$, Chun Sook Kim ${ }^{1}$ Kyu Sik Kang ${ }^{1}$, Sie Hyeon Yoo ${ }^{1}$, \\ Ji Won Chung ${ }^{1}$, Nan Seol Kim', and Seung Jin Lee ${ }^{2}$
}

Departments of ${ }^{1}$ Anesthesiology and Pain Medicine, ${ }^{2}$ Thoracic and Cardiovascular Surgery, Soonchunhyang University Hospital, College of Medicine, Soonchunhyang University, Cheonan, Korea

Surgical procedures necessitating the prolonged use of the lithotomy position can be associated with neuromuscular dysfunction. Compartment syndrome of the lower leg is a grave complication which, if unrecognized, can lead to either permanent neuromuscular dysfunction or limb loss. We report a case of compartment syndrome of lower leg that occurred in male patient aged 20 years after 380 minutes arthroscopic surgery in the lithotomy position. (Korean J Anesthesiol 2010; 59: S49-S52)

Key Words: Compartment syndrome, Lithotomy position, Neuromuscular dysfunction.

A prolonged operation in the lithotomy position can put pressure on the lower leg and lead to neuromuscular dysfunction. The leg compartment syndrome is a severe complication and it can cause permanent neuromuscular dysfunction or limb loss if it is not discovered early [1]. Although there are a few domestic reports of temporary leg dysfunction cases, such as common peroneal neropathy, caused by a long duration of operation in the lithotomy position $[2,3]$, it is a rare case where a permanent disorder was caused by a complete occlusion due to the thrombus in the popliteal artery and vein.

Herein, we report the case of the leg compartment syndrome at the opposite side of the operated part after a long duration of operation in the lithotomy position under general anesthesia. In addition, we provide a related literature survey.

\section{Case Report}

A $79 \mathrm{~kg}$, 20-year-old male patient was hospitalized in the orthopedic department for arthroscopic surgery at the right knee. He had no history of operation or venoocclusive disease and all the preoperative laboratory data were normal. As the preoperative treatment, glycopyrrolate $0.2 \mathrm{mg}$ and midazolam $3 \mathrm{mg}$ were injected into the muscle 30 minutes before the operation. After moving the patient to the operation room, blood pressure (BP), heart rate, and pulse oxygen saturation $\left(\mathrm{SpO}_{2}\right)$ were measured with a patient monitor (M1029A, Hewlett Packard Co., USA), and all were in the normal range.

For the induction of anesthesia, an intravenous injection of propofol $80 \mathrm{mg}$ and rocuronium $50 \mathrm{mg}$ and endotracheal

Received: April 16, 2010. Revised: April 28, 2010. Accepted: May 10, 2010.

Corresponding author: Ki Ryang Ahn, M.D., Department of Anesthesiology and Pain Medicine, Soonchunhyang University Hospital, College of Medicine, Soonchunhyang University, 23-20, Bongmyeong-dong, Cheonan 330-100, Korea. Tel: 82-41-570-2321, Fax: 82-41-573-3559, E-mail: ahnkiry@schmc.ac.kr

(c) This is an open-access article distributed under the terms of the Creative Commons Attribution Non-Commercial License (http:// creativecommons.org/licenses/by-nc/3.0/), which permits unrestricted non-commercial use, distribution, and reproduction in any medium, provided the original work is properly cited. 
intubation were conducted. The anesthesia was maintained with oxygen $2 \mathrm{~L} / \mathrm{min}$, nitrous oxide $2 \mathrm{~L} / \mathrm{min}$, and sevoflurane $2-3$ vol\%. The patient conditions were kept at BP 123/78 $\mathrm{mmHg}$, heart rate $72 / \mathrm{min}, \mathrm{SpO}_{2} 100 \%$, and end-tidal $\mathrm{CO}_{2}$ concentration $\left(\mathrm{EtCO}_{2}\right)$ 35-40 mmHg after 10 minutes.

As the preoperative preparation, the thigh and the knee at the opposite side of the operation were flexed by $30^{\circ}$ from the neutral position and fixed with a knee crutch. To prevent the muscle and nerve from being suppressed, sufficient pads were inserted. The operative part was in the foot drop position for the operation. The total operation time was 5 hours and 40 minutes, and the anesthetic time was 6 hours and 20 minutes. Physiological saline $3,000 \mathrm{ml}$ was intravenously injected during the operation, and the urine output was $1,000 \mathrm{ml}$. The intraoperative BP was well maintained and the operation was finished without any problem. The muscle relaxation was reversed with pyridostigmine $20 \mathrm{mg}$ and glycopyrrolate 0.4 $\mathrm{mg}$. The endotracheal tube was extubated after the recovery of spontaneous breathing and consciousness, and the patient was transported to the ward following the recovery in the recovery room.

Ten hours after the operation, the patient complained of a severe pain in the leg opposite to the operated one. A massive edema was found, and the patient made complaints of a severe pain during the palpation. In physical examination, foot drop, inability to evert the foot, hyperesthesia of the lateral and anterolateral aspects of the calf and medial half of the dorsum of the foot, weakness of plantar flexion of the foot and dorsalis pedis artery were not palpated. Dorsalis pedis artery was detectable only by examination with a Doppler echocardiography. Because it was judged that the compartment

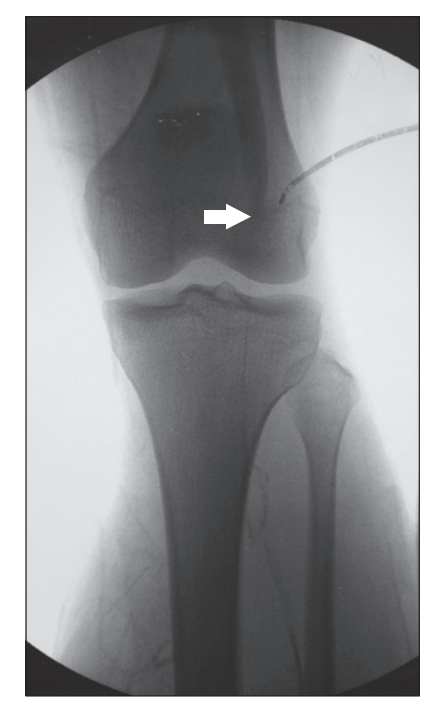

Fig. 1. Preoperative angiography shows complete occlusion of the popliteal artery. syndrome had already progressed considerably, an emergency fasciotomy was performed, without the compartment pressure measurement, under an epidural anesthesia with $0.5 \%$ bupivacaine. The muscle contraction, as well as the capillary filling and pulsation of the dorsalis pedis artery, and the posterior tibial artery were recovered. Twelve hours after the operation, the patient again complained of a severe pain, and the leg swelling became more serious. The popliteal arterial and venous occlusion were suspected, and the emergency angiography showed a complete occlusion at the popliteal artery (Fig. 1). Under general anesthesia, an intravenous injection of heparin 5,000 unit was carried out and the proximal portion was vascular clamped. After that, the thrombus was removed by an incision at the distal popliteal artery, and then, the restoration of the flow in the popliteal artery and posterior tibial artery was verified by the angiography (Fig. 2). In addition, a popliteal vein venotomy was conducted since venous occlusion was found from the ankle to the leg.

Urinalysis was performed to evaluate the renal function, and the result showed that the color was clear and the myoglobin was negative. The serum test results were creatine phosphokinase (CPK) $171 \mathrm{U} / \mathrm{L}$, blood urea nitrogen (BUN) $18 \mathrm{mg} / \mathrm{dl}$, creatinine $0.9 \mathrm{mg} / \mathrm{dl}$, and myoglobin $57 \mathrm{ng} / \mathrm{ml}$. The electrolytes were sodium $140 \mathrm{mmol} / \mathrm{L}$, potassium $4.5 \mathrm{mmol} /$ $\mathrm{L}$, and chloride $103 \mathrm{mmol} / \mathrm{L}$. The arterial blood gas analysis (ABGA) results were pH $7.43 \mathrm{mmHg}, \mathrm{PaCO}_{2} 41.3 \mathrm{mmHg}, \mathrm{PaO}_{2}$ $274.0 \mathrm{mmHg}$, and the base excess $-1.4 \mathrm{mmol} / \mathrm{L}$, all being in the normal range. The physical examination 10 days after the operation showed that the function of the posterior tibial nerve and the common peroneal nerve was missed. The small necrotic part of the tibialis anterior muscle was debrided
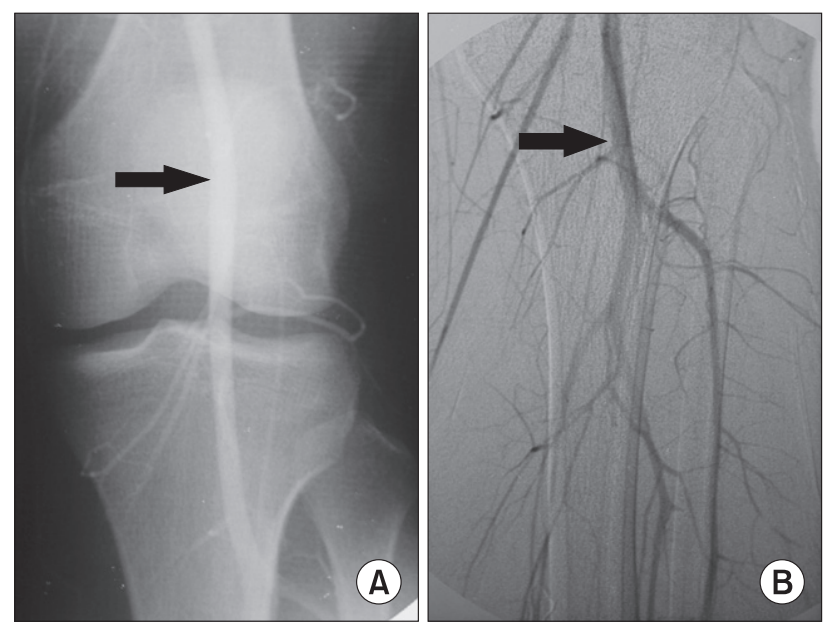

Fig. 2. Postoperative angiography shows restoration of flow in the popliteal artery (A) and posterior tibial artery (B) after successful recanalization. 
and skin grafted. The CPK, BUN, creatinine, myoglobin, and electrolyte test results were all in the normal range.

Electrodiagnosis was performed 20 days after the operation. No complex muscle action potential (CMAP) in the peroneal nerve and tibial nerve was observed by the motor nerve conduction velocity (MNCV). Sensory nerve action potential (SNAP) was not found at the superficial peroneal nerve and sural nerve by the sensory nerve conduction velocity (SNCV). The electromyography (EMG) showed abnormal spontaneous potentials at the anterior tibial muscle, gastrocnemius muscle, extensor hallucis longus muscle, and extensor digitorum brevis muscle, but no motor unit action potential (MUAP) at the hallucis muscle and extensor digitorum brevis muscle, which indicated that the function of the superficial and deep peroneal nerve and tibial nerve was lost. Physiotherapy and electrodiagnosis tests were repeated many times during the hospital stay; the tests showed that symptoms were not improved and the muscle atrophy continuously progressed. The patient was discharged after 7 months of hospitalization. A psychiatric disorder was caused after the discharge by a permanent motor abnormality, and the patient is currently being treated in the psychiatry department, as he is hopeless.

\section{Discussion}

Lithotomy is the position that is frequently taken in many kinds of operations. Postoperative compartment syndrome, rhabdomyolysis, and neuropathy of the lower extremity are caused in this position, regardless of the kind of operations [46], and the clinical symptoms, such as extreme pain unrelieved by analgesics, paresthesias, loss of sensation, intact pulse, and tense swelling of the involved region, are found [7].

The pathogenesis of the compartment syndrome includes the perfusion disorder by the direct local pressure on the lower extremity as the initiating event, transudation and exudation by the necrosis of muscle and loss of capillary wall integrity, and massive edema within the compartment. The edema increases the pressure within the compartment and affects the vascular supply, and repetition of this process forms the vicious cycle [8].

The reason why the compartment syndrome takes place in only some patients is not clearly known. In general, the reasons have been reported as: 1) systemic hypotension and loss of driving pressure to the extremity (augmented by elevation of the extremity); 2) vascular obstruction of major leg vessels by intrapelvic retractors, by excessive flexion of knee or hip, or by undue popliteal pressure from a knee crutch; and 3) external compression of the elevated extremity by straps or leg wrappings that are too tight, by the inadvertent pressure of the leg of a surgical assistant, or by the weight of the extremity against a poorly supportive leg holder [9]. In this case report, the intraoperative blood pressure was well maintained; the knee was not excessively flexed during the positional change; and a sufficient amount of pads were applied to reduce the pressure. However, the position of the leg was fixed unnecessarily high above the operation table, which might be the cause for the vascular occlusion due to the formation of thrombus in the popliteal artery and vein by putting pressure on the popliteal fossa. External pressure that might have been engaged by an operation assistant could be a cause, though it is uncertain whether or not pressure was actually engaged.

Other risk factors that might cause the compartment syndrome in the lithotomy position are age $>70$, body mass index $(\mathrm{BMI})<20$, the patient's history of the risk of nerve ischemia or neuropathy, a history of smoking, diabetes, vascular disease, connective disease, anemia, nerve deficit, or spinal pathology [6], and the operation duration. The maximum safe time length of the operation has not been clearly known, but it was reported that the compartment syndrome occurred after more than 4 hours of operation by Warner and Martin [9], 10-12 hours of operation by Lampert et al. [10], and 13 hours and 15 minutes of operation by Kumamato et al. [11]. These risk factors were not involved in the case report herein, but the operation took 6 hours and 20 minutes. Such a long duration of operation might have formed thrombus in the popliteal artery and vein and caused the compartment syndrome by engaging a continued pressure on the leg.

Complications of the compartment syndrome include permanent or transient neuromuscular deficits and rhabdomyolysis with or without renal failure. Rhabdomyosis is the cause of skeletal muscle injury and acute failure is found $2-5 \%$ of rhabdomyosis patients [12-14]. In this case report, urinalysis and a serum test were performed to prevent rhabdomyolysis or acute renal failure. The postoperative urinalysis showed no myoglobin, and the serum test showed that CPK, BUN, and creatinine were in the normal range. In addition, no electrolyte or acid-base disturbance was found. The reason why acute renal failure or rhabdomyolysis did not take place in this case report is not accurately understood, but the patient did not have the risk factors, such as crush injuries, severe infection, alcohol intake, and drug toxicity, that might cause rhabdomyolysis in higher probability, and the vessel was influenced only by the long duration of pressure.

Since irreversible neuromuscular defects occur 12-24 hours after the operation [10], diagnosis is very important. Mar et al. [15] reported that the pain is unreliable, as cardinal symptom and physical examination are also unreliable for the diagnosis. Mar et al. [15] also reported that patient-controlled analgesia opioids and regional analgesia do not matter in the diagnosis of the compartment syndrome, if only they are adequately monitored. Thus, they commented that continuous patient 
monitoring and measurement of the compartment pressure are required for an accurate diagnosis, regardless of the type of analgesia and the high index of clinical suspicion. The reason for the permanent abnormality in this case may be that it occurred not in the operated part but the opposite part, which was observed 10 hours after the operation when the patient complained of severe pain. Lack of continuous patient monitoring and the compartment pressure measurement might have caused the neuromuscular dysfunction.

In the case of operation in the lithotomy position, the fixing duration should be reduced in order to prevent postoperative neurologic complications. It has been known that the risk of motor neuropathy is increased by 100 times as the fixing time increases by 1 hour [6]. Therefore, the lithotomy position should be taken during the operation only when it is necessary, and a position change must be carried out if the operation time is long.

In conclusion, to avoid compartment syndrome in an operation performed in the lithotomy position, 1) the intraoperative blood pressure should not be significantly reduced; 2) the factors of external pressure should be avoided by the use of sufficient pads; 3 ) the leg position should be kept at the atrium level; 4) the duration of the operation should be reduced as much as possible, and 5) the patient should be monitored continuously.

\section{References}

1. Riber C, Flink PO, Svendsen LB, Wøjdemann M. Compartment syndrome as a postoperative complication of lithotomy position. Ugeskr Laeger 1995; 157: 4576-7.

2. So KY, Han HC, Kim CS, Chung CD, Yu BS. Common peroneal nerve palsy after lithotomy position: two case reports. Korean J Anesthesiol 2004; 46: 250-2.
3. Kim HJ, Kim SJ. Common peroneal neuropathy after surgery in the lithotomy position: a case report. Anesth Pain Med 2009; 4: 79-82.

4. Beraldo S, Dodds SR. Lower limb acute compartment syndrome after colorectal surgery in prolonged lithotomy position. Dis Colon Rectum 2006; 49: 1772-80.

5. Bagley WH, Yang H, Shah KH. Rhabdomyolysis. Intern Emerg Med 2007; 2: 210-8.

6. Warner MA, Warner DO, Harper CM, Schroeder DR, Maxson PM. Lower extremity neuropathies associated with lithotomy positions. Anesthesiology 2000; 93: 938-42.

7. Dutton RP, McCunn M. Anesthesia for trauma. In: Miller's Anesthesia. 6th ed. Edited by Miller RD: Philadelphia, ChurchillRivingstone. 2005, pp 2451-95.

8. Krarup PM, Rawashdeh YF. Lower limb compartment syndrome subsequent to surgery in the lithotomy position. Ugeskr Laeger 2008; 170: 1539-43.

9. Warner MA, Martin JT. Patient position. In: Clinical Anesthesia. 4th ed. Edited by Barash PG, Cullen BF, Stoelting RK: Philadelphia, Lippincott Williams \& Wilkins. 2001, pp 639-65.

10. Lampert R, Weih EH, Breucking E, Kirchhoff S, Lazica B, Lang K. Postoperative bilateral compartment syndrome resulting from prolonged urological surgery in lithotomy position. Serum creatine kinase activity (CK) as a warning signal in sedated, artificially respirated patients. Anaesthesist 1995; 44: 43-7.

11. Kumamoto T, Hara S, Yoshitake A, Shimoda O, Terasaki H. Well leg compartment syndrome during prolonged surgery in the lithotomy position. Masui 2007; 56: 695-8.

12. West H. Rhabdomyolysis associated with compartment syndrome resulting in acute renal failure. Eur J Emerg Med 2007; 14: 368-70.

13. Ho GH, van Laarhoven CJ, Ottow RT. Compartment syndrome in both lower legs after prolonged surgery in the lithotomy position. Ned Tijdschr Geneeskd 1998; 142: 1210-2.

14. Polderman KH. Acute renal failure and rhabdomyolysis. Int J Artif Organs 2004; 27 : 1030-3.

15. Mar GJ, Barrington MJ, McGuirk BR. Acute compartment syndrome of the lower limb and the effect of postoperative analgesia on diagnosis. Br J Anaesth 2009; 102: 3-11. 